\title{
Case Report and Review of the Literature
}

\section{Intraarterial Injection of Drugs of Abuse into Femoral Artery: Case Report and Review of the Literature}

\author{
Nina Kobilica* and Vojko Flis
}

Department of Vascular Surgery, Surgical Clinics, University Clinical Centre Maribor, Slovenia

\begin{tabular}{l} 
A R T I C L E I N F O \\
\hline Article history: \\
Received: 12 January, 2021 \\
Accepted: 28 January, 2021 \\
Published: 11 February, 2021 \\
\hline Keywords: \\
Drug abuse \\
intraarterial injection \\
amputation
\end{tabular}

\begin{abstract}
A B S T R AC T
Accidental intraarterial injection is a serious condition which can compromise the viability of the limb within hours. There are no evidence based guidelines suggesting the proper treatment protocol and there is no consensus about the ideal treatment for these events. We present a case where a mixture of benzodiazepine pills and street heroin was injected in right femoral artery. Patient arrived in hospital days after event. With intraarterial application of thrombolysis, vasodilators and heparin major amputation was avoided. It seems that in selected cases combined therapy with rtPA lysis and PGE1 intra-arterial infusion may prevent major limb amputation even in delayed presentation of acute leg ischaemia caused by inadvertent injection of drugs of abuse.
\end{abstract}

\section{Introduction}

Intravenous drug abuse is a global social and health care problem. It is estimated that there are approximately 13.2 intravenous- drug addicts worldwide [1]. Morbidity and mortality are related to the effect of substances themselves and to the risks intrinsic in the practice [2]. In terms of vascular injury, the lower limbs are most commonly affected [3]. Injection drug users traditionally use veins for drug delivery but arteries are occasionally used intentionally or inadvertently. Owing to the clandestine nature of intravenous drug abuse femoral region is often used as an access door to venous system [4]. Accidental intra-arterial injection of drugs in common femoral artery is a sporadic complication in intravenous- drug addicts. This complication, although well documented, is relatively rare and the series of case reports are small. The clinical picture depends on the drug properties and ranges from partial ischaemia to necrosis of affected extremity [5]. Limb ischaemia and subsequent tissue necrosis in drug abusers has been widely reported after intra-arterial injection of a number of substances including heroin and benzodiazepines tablets [6]. Often intra-arterial injections of crushed, dissolved tablets containing different sedatives or narcotics and multiple constituents, such as microcrystalline cellulose or magnesium stearate are used. Additionally, the injections are invariably contaminated with impurities when procured illegally and there is no generally accepted treatment algorithm to treat critical ischaemia after intra-arterial drug injection [7]. Early diagnosis of the ischaemic syndrome followed by an immediate therapy is essential for limb rescue, however in drug abusers time is usually lost until proper diagnosis and effective therapy. We report a case in which a mixture of drugs was injected into the right femoral artery and patient arrived few days later with acute ischaemia of the right leg at our emergency department.

\section{Case Report}

A 20-year-old woman, drug addict, went to emergency department for a right foot pain. She described an onset of continuous pain after injection of a mixture of street heroin and benzodiazepine pills into her right groin that occurred few days earlier. She managed to diminish the pain with repetitious heroin injections and decided to visit physician only when she detected the change of finger colour of right foot. Examination revealed swollen right foot, with dark fingers and plantar part of foot. The skin of the lower leg was marbled and livid with large areas of superficial necrosis (Figures $1 \& 2$ ). Femoral, popliteal and pedal pulses were palpable. There was a pain in motion and absence of capillary refill. An angiogram showed interruption of flow in plantar arcs and all digital arteries (Figure 3).

${ }^{*}$ Correspondence to: Nina Kobilica, Department of Vascular Surgery, Surgical Clinics, University Clinical Centre Maribor, Slovenia; E-mail: nina.kobilica@gmail.com

(C) 2021 Nina Kobilica. This is an open-access article distributed under the terms of the Creative Commons Attribution License, which permits unrestricted use, distribution, and reproduction in any medium, provided the original author and source are credited. Hosting by Science Repository. http://dx.doi.org/10.31487/j.SCR.2021.02.12 


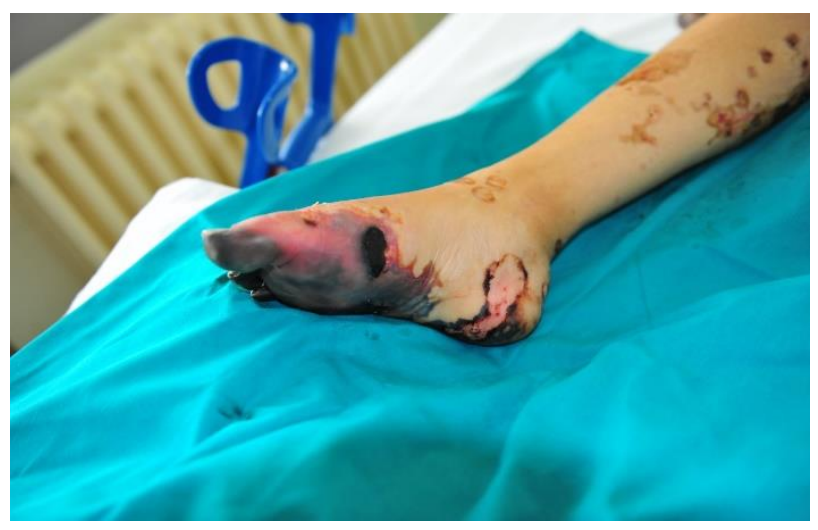

Figure 1: Median view of the affected foot at the admission to hospital.

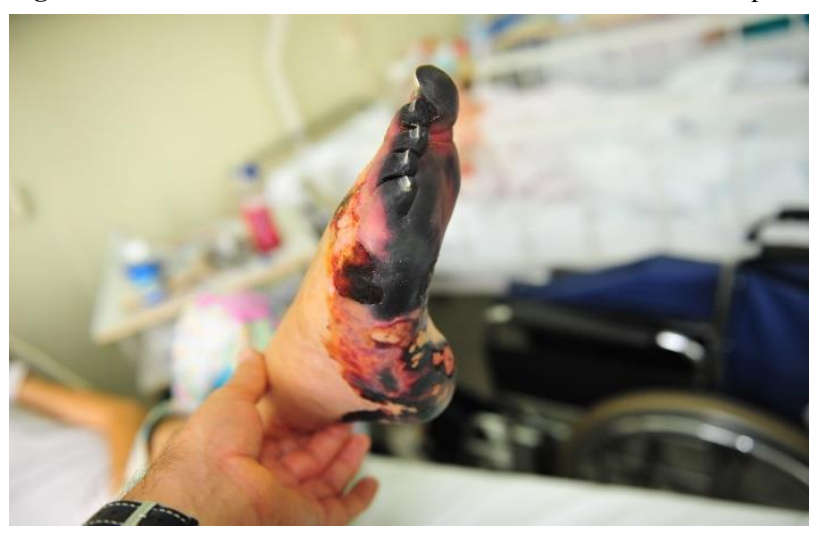

Figure 2: Lateral view of the affected foot at admission to hospital.

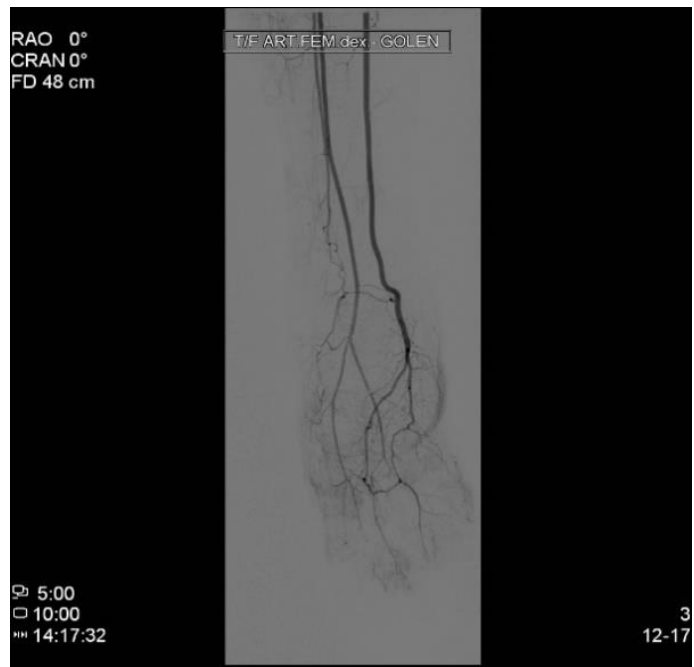

Figure 3: DSA of crural arteries showed obliterated pedal arches and digital arteries.

The patient was admitted to the intensive care unit and intra-arterial thrombolysis with rtPA was started (100mg in three hours). Heparin was infused at the rate of $500 \mathrm{IU} / \mathrm{h}$. Additionally continuous nitroglycerine $(2 \mathrm{mg} / \mathrm{h})$ and prostaglandin E1 intra-arterial infusion (2x20 microg/d) was applied. Drug withdrawal was treated with methadone. Over the course of next six days the patient condition improved, the lower leg showed adequate perfusion and intra-arterial infusions of prostaglandin and nitroglycerine were discontinued. Although initially bellow knee amputation was considered eventually transmetatarsal amputation was required and the rest of the leg was spared.

\section{Discussion}

Accidental or intentional arterial drug injection can cause a wide range of vascular complications, from simple vascular irritation to severe infections, thrombosis and gangrene. Most commonly upper extremity is involved, however there are occasional case reports of lower extremity injuries mainly in clandestine drug addicts $[5,8]$. Injecting drug via femoral vein puncture has been identified as more widespread than previously recognized and it is associated with considerable morbidity $[4,9]$. Repeated injecting might cause vascular lesions, which affect both veins and arteries. Acute limb ischaemia as a consequence of arterial injection of drugs is well recognized [3]. Underlying pathological changes resulting in ischaemia are not completely understood but include chemical arteritis due to injection of irritant substances resulting in endothelial damage and swelling. This in turn causes arterial, venous and capillary thrombosis secondary to platelet aggregation and injury from inflammatory mediators $[3,10]$. Investigations are limited but angiography should be considered to ascertain the extent of injury and allow for an intra-arterial catheter to be left in situ for infusions and subsequent control angiography if desired [3].

Various forms of medical treatment have been advocated including vasodilators, heparin and thrombolysis but with varied responses $[3,5$, $7,11]$. Haemodilution with low molecular weight dextran, continuous plexus blocks and regional nerve blocks for sympathicolysis and pain control, and corticosteroids were also applied, whereas sympathetic antagonists and hyperbaric oxygen therapy combined with antiplatelet agents have rarely been used [7, 11-13]. A literature search revealed that a generally accepted treatment algorithm for intra-arterial drug injection to stop critical ischaemia is missing $[3,7,11]$. Only case studies on the treatment of inadvertent injection of various drugs have been published so far $[3,5]$. The small number of patients involved in most of empiric studies made it difficult to develop an effective and well-accepted treatment protocol. Only report from Rohm and co-authors includes an unusually high number of patients with inadvertent intra-arterial injection in lower extremity [7].

Rohm and co-authors report on the good clinical outcome of 16 drug abusers (six of them with femoral artery injection, rest were upper extremity injections) who developed critical limb ischaemia after inadvertent intra-arterial injection of crushed, dissolved flunitrazepam tablets and were treated with an intensive protocol combining immediate anticoagulation with heparin, long lasting local thrombolysis and vasodilatation [7]. It is believed that combination of prostaglandin E1 (PGE1) and rt-PA stops the pathophysiological cascade and ischaemic process [7]. Prostaglandin E1 enhances the fibrinolytic efficacy of rt-PA and does so, in part, by inhibiting platelet aggregation [14, 15]. Rohm and co-authors reported good therapeutic outcome with combined therapy (thrombolysis, PGE1] even in patients who were admitted more than 24 hours after accidental injection [7].

Our patient was admitted several days after intra-arterial drug injection. Although gangrene of foot with large ischaemic skin areas of lower leg were present and below knee amputation was initially considered combined intra-arterial therapy (rt-PA lysis and PGE1 infusion) improved the lower leg perfusion and allowed for minor amputation only. 


\section{Conclusion}

There is no standardized treatment protocol for intra-arterial injection of drugs of abuse. Only few cases have been published concerning the lower extremity. Different options make the right therapeutic decision very difficult because the choice of suitable treatment is dependent on the individual case and the experience of involved physicians. Often outcome is disastrous with the loss of parts of the limb. However, it seems that combined therapy with rt-PA lysis and PGE1 intra-arterial infusion may prevent major limb amputation even in delayed presentation of acute leg ischaemia caused by inadvertent injection of drugs of abuse.

\section{REFERENCES}

1. Aceijas C, Stimson GV, Hickam M, Rhodes T, United Nation Reference Group on HIV/AIDS Prevention and care among IDU in Developing and Transitional Countries (2004) Global overview of injecting drug users. AIDS 18: 2295-2303. [Crossref]

2. D'Ovidio C, Vellante P, Constantini S, Carnevale A (2013) Death due to an unrecognized groin abscess in a drug addict: a retrospective study. J Foensic Legal Med 20: 382-386. [Crossref]

3. Fiddes R, Khatttab M, Abu Dakka M, Al Khaffaf H (2010) Patterns and management of vascular injuries in intravenous drug users: a literature review. Surgeon 8: 353-361. [Crossref]

4. Koželj M, Kobilica N, Flis V (2006) Infected femoral pseudoaneurysms from intravenous drug abuse in young adults. Wien klin Wochenschr 118: 71-75. [Crossref]

5. Betz T, Steinbauer M, Uhl C, Toepl I (2011) Necrosis of the leg after intraarterial drug injection. Vasa 40: 163-166. [Crossref]
6. Stonebridge PA, Callam MJ, Farouk M, Muire JA (1990) Intra-arterial injection of oral medication in HIV positive drug addicts. Br J Surg 77: 333-334. [Crossref]

7. Rohm S, Staab H, Schulz H, Richter O, Aust G (2014) Good clinical outcome after accidental intra-arterial injection of fluzepam tablets in 16 drug abusers with critical limb ischemia. Eur J Vasc Endovasc Surg 47: 61-67. [Crossref]

8. Devulapalli C, Han KD, Bello RJ, LaPorte DM, Hepper CT et al. (2015) Inadvertent intra-arterail drug injections in the upper extremity: systematic review. J Hand Surg Am 40: 2262-2268. [Crossref]

9. Senbajo R, Strang J (2011) The needle and the damage done: clinical and behavioural markers of severe femoral vein damage among groin injectors. Drug Alcohol Depend 119: 161-165. [Crossref]

10. Calder KK, Severyn FA (2003) Surgical emergencies in the intravenous drug user. Emerg Med Clin North Am 21: 1089-1116. [Crossref]

11. Hohenstein C, Herdtle S, Hoyme M, Chaudhary T (2014) Rescue of the limb after accidental injection of diazepam into femoral artery. Am J Emerg Med 32: 1149.e5-1149.e6. [Crossref]

12. Treiman GS, Yellin AE, Weaver FA, Barlow WE, Treiman RL et al. (1990) An effective treatment protocol for intraarterial drug injection. $J$ Vasc Surg 12: 456-465. [Crossref]

13. Adir Y, Halpern P, Nachum Z, Bitterman H (1991) Hyperbaric oxygen therapy for ischaemia of the hand due to intra-arterial injection of methadone and flunitrazepam. Eur J Vasc Surg 5: 677-679. [Crossref]

14. Vaughan DE, Plavin SR, Schafer AI, Loscazo J (1989) PGE1 accelerates thrombolysis by tissue plasminogen activator. Blood 73 : 1213-1217. [Crossref]

15. Alstaedt HO (1999) Experience with the therapy of acute finger ischemia by high-dose intra-arterial infusion of PGE1 thorugh the surgically introduced cathether. Chirurg 70: 1144-1148. [Crossref] 